\title{
Classification of short-term complications after transanal endorectal pullthrough for Hirschsprung's disease using the Clavien-Dindo-grading system
}

\author{
Nils Hoff ${ }^{1} \cdot$ Tomas Wester $^{1} \cdot$ Anna Löf Granström ${ }^{1,2}$
}

Accepted: 6 August 2019 / Published online: 14 August 2019

(c) The Author(s) 2019

\begin{abstract}
Purpose Hirschsprung's disease (HSCR) is a developmental defect of the enteric nervous system. Transanal endorectal pullthrough (TERPT) is one of the surgical procedures for HSCR. Clavien-Dindo is an objective classification system, used worldwide, to describe postoperative complications. The aim of this study was to use Clavien-Dindo grading for short-term complication after TERPT.

Methods This was a cohort study including all 69 individuals, with biopsy-verified HSCR, managed with TERPT at our institution between 2006 and 2018. Data on the surgical procedure, as well as short-term complications, were retrieved from the medical records. The main outcome was postoperative complications graded according to Clavien-Dindo.

Results Fifteen (22\%) of the 69 patients (51 males) had a short-term postoperative complication graded according to ClavienDindo. The complications were Grade I in ten patients, Grade II in four patients, and Grade IIIb in one patient. Individuals with a Clavien-Dindo complication had a significantly longer post-operative hospital stay [median 6 days (4-30) compared to 4 days $(1-22), p=0.035$ ].

Conclusions It is important to describe postoperative complications in a structured way to make it possible to compare studies. Post-operative complications, according to Clavien-Dindo, occurred in $22 \%$ of the patients after TERPT.
\end{abstract}

Keywords Hirschsprung disease $\cdot$ Transanal endorectal pultthrough $\cdot$ Clavien-Dindo $\cdot$ Complications

\section{Introduction}

Hirschsprung's disease (HSCR) is a congenital malformation, characterized by the absence of ganglion cells in the distal hindgut. The absence of ganglion cells results in absent peristalsis in the affected bowel and the development of functional intestinal obstruction. HSCR is the most common congenital motility disorder in children, affecting 1 in 5000 live births [1]. HSCR is treated with rectosigmoid resection and coloanal anastomosis, using normally innervated colon for the anastomosis to the anus. In 1998, De la

Anna Löf Granström

anna.lof@ki.se

1 Department of Women's and Children's Health, Karolinska Institutet, Stockholm, Sweden

2 Division of Pediatric Surgery, Astrid Lindgren Children's Hospital, S3:02, Karolinska University Hospital, Solna, SE-17176 Stockholm, Sweden
Torre described transanal endorectal pullthrough (TERPT). Modifications of this technique are frequently used today [2]. Short-term complications of TERPT have previously been described. Anastomotic leakage is the most serious early postoperative complications reported to occur in $0-1.5 \%$ of the patients [3, 4]. Anastomotic stricture, postoperative Hirschsprung-associated enterocolitis (HAEC), wound infections, bleeding, and perianal excoriations are other early post-operative complications $[3,4]$. To be able to compare post-operative outcomes between different surgical centres, there is a need for an objective grading system of the early complications. Clavien-Dindo is a grading system used to objectively and reproducibly classify postoperative complications [5]. It consists of seven grades with increasing severity. Grade I being the most benign, stating that any deviation from the normal postoperative course without pharmacological treatment or surgical, endoscopic, and radiological interventions and Grade V is death (Table 1).

The aim of this study was to use Clavien-Dindo grading for short-term complication after TERPT and to investigate 
Table 1 Clavien-Dindo classification of surgical complications

\begin{tabular}{|c|c|}
\hline Grade & Definition \\
\hline Grade I & $\begin{array}{l}\text { Any deviation from the normal postoperative course without the need for pharmacological treatment or surgical, endoscopic, and } \\
\text { radiological interventions } \\
\text { Allowed therapeutic regimens are: drugs as antiemetics, antipyretics, analgetics, diuretics, electrolytes, and physiotherapy. This grade } \\
\text { also includes wound infections opened at the bedside }\end{array}$ \\
\hline Grade II & $\begin{array}{l}\text { Requiring pharmacological treatment with drugs other than such allowed for grade I complications. Blood transfusions and total } \\
\text { parenteral nutrition are also included }\end{array}$ \\
\hline Grade III & Requiring surgical, endoscopic or radiological intervention \\
\hline IIIa & Intervention not under general anesthesia \\
\hline IIIb & Intervention under general anesthesia \\
\hline Grade IV & Life-threatening complication (including CNS complications) ${ }^{\mathrm{a}}$ requiring IC/ICU management \\
\hline IVa & Single organ dysfunction (including dialysis) \\
\hline $\mathrm{IVb}$ & Multiorgan dysfunction \\
\hline Grade V & Death of a patient \\
\hline Suffix "d" & $\begin{array}{l}\text { If the patient suffers from a complication at the time of discharge, the suffix "d" (for disability") is added to the respective grade of } \\
\text { complication. This label indicates the need for a follow-up to fully evaluate the complication }\end{array}$ \\
\hline
\end{tabular}

Presented as the description from: Classification of Surgical Complications, A New Proposal With Evaluation in a Cohort of 6336 Patients and Results of a Survey [5]

$C N S$ central nervous system, $I C$ intermediate care, $I C U$ intensive care unit

${ }^{a}$ Brain hemorrhage, ischemic stroke, subarachnoidal bleeding, but excluding transient ischemic attacks

risk factors for developing a Clavien-Dindo short-term complication.

\section{Methods}

This was a cohort study of patients who underwent surgery for HSCR between 2006 and 2018 at the Department of Pediatric Surgery, Karolinska University Hospital, Stockholm, Sweden. Patients with HSCR, confirmed with histology, were identified and included in the study. Data on HSCR and the surgical management were collected from the medical records. Exclusion criteria were patients who underwent other surgical procedures than TERPT including patient with total colonic aganglionosis (TCA). The followup period of the patients included the first 30 days after TERPT. Retrospective grading according to Clavien-Dindo was performed for each case as well as every type of complication that was registered (anastomotic leakage, stricture, postoperative HAEC, wound infections, and bleeding). Stricture was defined as a stricture treated with dilatation under general anesthesia.

\section{Statistical analysis}

Categorical data were presented as frequencies or proportions and analyzed with two-tailed Fisher's exact test. Numerous data were presented as mean and standard deviation if normally distributed, otherwise as medians and range. Continuous data were analyzed with $t$ test for normally distributed data and Mann-Whitney $U$ test for non-normally distributed data. Categorical variables were compared using Chi-square test. In all tests, $p<0.05$ was considered statistically significant. Univariable logistic regression analyses were used to calculate the specific risk factors presented as odds ratio (OR) with the confidence interval (CI) set to $95 \%$.

Statistical analyses were executed using IBM SPSS Statistics for Windows, version 25 (IBM Corp., Armonk, NY, USA) and MedCalc Statistical Software version 18.2.1 (MedCalc Software bvba, Ostend, Belgium; https://www. medcalc.org; 2016).

\section{Results}

\section{Demographic data}

During the time period from April 2006 until April 2018, 89 patients with HSCR managed at the Department of Pediatric Surgery of Karolinska University Hospital were identified. Twenty of these patients were excluded, six had undergone another surgical procedure, seven had TCA, three had tundergone TERPT at another institution, and four had HSCR, but had not yet undergone the surgical procedure. Sixty-nine (51 male) were finally included in the study. The median age at TERPT was 62 days (range 10-3355). Associated malformations were found in nine patients (13\%). Ventricular septum defects were the most common associated malformation. Eight (11.6\%) individuals had a syndrome, seven of whom had Down's syndrome. Four individuals (5.8\%) 
had HAEC before TERPT and ten patients (14.5\%) had a stoma before TERPT. Demographic data are summarized in Table 2.

\section{Complication within 30 days of TERPT: Clavien- Dindo}

Patients who had postoperative complications within 30 days of TERPT were graded using the Clavien-Dindo grading system. A total of $15(22 \%)$ patients had a postoperative complication, ten of whom (14.4\%) had a Grade I complication, four (5.8\%) a Grade II, and $1(1.4 \%)$ a Grade IIIb complication. All ten Grade I, complications were related to postoperative pain management. Three of the patients had an epidural catheter complication and seven of the patients received opiates not in compliance with a normal postoperative course. Four patients had a Grade II complication, one of whom required total parenteral nutrition, one had seizures and needed benzodiazepine, one had a superficial wound infection and required antibiotics, and one required blood transfusion post-operatively. One patient had a Grade IIIb complication caused by an abscess, which later needed a re-operation with an ileostomy. No death was reported.

Individuals with a Clavien-Dindo classified complication had a significantly longer postoperative hospital stay, median 6 days (range 4-30) compared to individuals without complications who had a median postoperative stay of 4 days (range $1-22$ ), $p=0.035$. One patient without a complication had a postoperative stay of 35 days due to Down's syndrome with a severe congenital heart defect and heart failure and was excluded from the analysis of postoperative stay, as he was considered an outlier. The median postoperative hospital stay was 5 days (range 1-35). Eleven patients (15.9\%) were readmitted within 30 days of TERPT and one patient was re-operated within 30 days of TERPT due to an abscess formation, as shown in Table 3.

\section{Risk factors for short-term complications}

We found that there were no specific risk factors for developing a Clavien-Dindo short-term complication. All variables are shown in Table 4.

\section{Discussion}

This is the first study that describes post-operative complications in patients with Hirschsprung's disease graded according to Clavien-Dindo. A Clavien-Dindo complication was found in 15 (22\%) of the patients. The purpose of this study was to highlight all possible adverse events after TERPT, such as inappropriate postoperative analgesia as well as prolonged time to oral feeding. To be able to compare results

Table 2 Demographic data of the study population

\begin{tabular}{|c|c|c|c|c|c|}
\hline & $\begin{array}{l}\text { Total } \\
n=69\end{array}$ & $\begin{array}{l}\text { Female } \\
n=18\end{array}$ & $\begin{array}{l}\text { Male } \\
n=51\end{array}$ & Missing data & $p$ value \\
\hline Gender $n(\%)$ & $69(100)$ & $18(26.1)$ & $51(73.9)$ & 0 & \\
\hline Associated malformations $n(\%)$ & $9(13)$ & $1(5.6)$ & $8(15.7)$ & 0 & 0.428 \\
\hline Syndrome $n(\%)$ & $8(11.6)$ & $0(0)$ & $8(15.7)$ & 0 & 0.101 \\
\hline Down's syndrome $n(\%)$ & $7(10.1)$ & $0(0)$ & $7(13.7)$ & 0 & 0.203 \\
\hline Heredity $n(\%)$ & $9(13)$ & $6(33.3)$ & $3(5.9)$ & 0 & 0.008 \\
\hline Gestational age (weeks), median (range) & $39(30-42)$ & $38(30-42)$ & $39(31-42)$ & $14(5 \mathrm{~F} / 9 \mathrm{M})$ & 0.779 \\
\hline Birth weight, median, g (range) & $3421(1335-4500)$ & $3200(1335-4100)$ & $3422(1854-4500)$ & $11(5 \mathrm{~F} / 6 \mathrm{M})$ & 0.328 \\
\hline \multicolumn{6}{|l|}{ Initial symptoms $n(\%)$} \\
\hline Neonatal intestinal obstruction & $58(84.1)$ & $13(72.2)$ & $45(88.2)$ & 0 & 0.140 \\
\hline HAEC & $0(0)$ & $0(0)$ & $0(0)$ & 0 & \\
\hline Chronic constipation & $11(15.9)$ & $5(27.8)$ & $6(11.8)$ & 0 & \\
\hline HAEC preop $n(\%)$ & $4(5.8)$ & $1(5.6)$ & $3(5.9)$ & 0 & 1.000 \\
\hline Age at first rectal biopsy (days), median (range) & $9.5(2-3082)$ & $13(2-1067)$ & $9(3-3082)$ & $5(2 \mathrm{~F} / 3 \mathrm{M})$ & 0.378 \\
\hline Age at TERPT (days), median (range) & $62(10-3355)$ & $66.5(17-1158)$ & $60(10-3355)$ & 0 & 0.477 \\
\hline \multicolumn{6}{|l|}{ Level of aganglionosis $n(\%)$} \\
\hline Rectum/rectosigmoid junction & $61(8.4)$ & $15(83.3)$ & $46(90.2)$ & 0 & 0.421 \\
\hline Long segment (proximal to left flexure) & $8(10.7)$ & $3(15)$ & $5(9.1)$ & 0 & \\
\hline Preoperative stoma $n(\%)$ & $10(14.5)$ & $3(16.7)$ & $7(13.7)$ & 0 & 0.713 \\
\hline
\end{tabular}

Significant result is in bold

Missing data shown under missing data, in total and how many females (F) and males (M) were missing, respectively HAEC Hirschsprung-associated enterocolitis, TERPT transanal endorectal pullthrough 
Table 3 Complications within 30 days of TERPT, in total, female and male

\begin{tabular}{lcccc}
\hline & $\begin{array}{l}\text { Total } \\
N=69\end{array}$ & $\begin{array}{l}\text { Female } \\
n=18\end{array}$ & $\begin{array}{l}\text { Male } \\
n=51\end{array}$ & $p$ value \\
\hline Postoperative complication <30 days of TERPT $n(\%)$ & $15(21.7)$ & $2(11.1)$ & $13(25.5)$ & 0.321 \\
Clavien-Dindo classification $n(\%)$ & & & & \\
Grade 0 & $54(78.3)$ & $16(88.9)$ & $38(74.5)$ & 0.599 \\
Grade I & $10(14.5)$ & $1(5.6)$ & $9(17.6)$ & \\
Grade II & $4(5.8)$ & $1(5.6)$ & $3(5.9)$ & \\
Grade IIIb & $1(1.4)$ & $0(0)$ & $1(2)$ & \\
Anastomotic leakage $n(\%)$ & $0(0)$ & $0(0)$ & $0(0)$ & - \\
Stricture $n(\%)$ & $12(17.4)$ & $1(5.6)$ & $11(21.6)$ & 0.163 \\
Postoperative HAEC $n$ (\%) & $23(33.3)$ & $3(16.7)$ & $20(39.2)$ & 0.144 \\
Bleeding $n$ (\%) & $1(1.4)$ & $0(0)$ & $1(2)$ & 1.0 \\
Wound infection $n$ (\%) & $2(2.9)$ & $0(0)$ & $2(3.9)$ & 1.0 \\
Postoperative hospital (days), median (range) & $5(1-35)$ & $4.5(1-10)$ & $5(2-35)$ & 0.593 \\
Readmission within 30 days of TERPT $n(\%)$ & $11(15.9)$ & $2(11.1)$ & $9(17.6)$ & 0.715 \\
Reoperation within 30 days of TERPT $(\%)$ & $1(1.4)$ & $0(0)$ & $1(2)$ & 1.0 \\
\hline
\end{tabular}

HAEC Hirschsprung-associated enterocolitis, TERPT transanal endorectal pullthrough
Table 4 Risk factors for developing a Clavien-Dindo classified shortterm complication

\begin{tabular}{ll}
\hline & Univariate OR (95\% CI) \\
\hline $\begin{array}{l}\text { Clavien-Dindo } \\
\text { Male gender }\end{array}$ & $0.37(0.05-1.53)$ \\
Associated malformation & $2.00(0.38-8.82)$ \\
Syndrome & $1.23(0.17-6.11)$ \\
Heredity & $0.41(0.02-2.53)$ \\
Preoperative HAEC & $1.21(0.06-10.35)$ \\
Preoperative stoma & $2.91(0.65-12.06)$ \\
Age at TERPT days & $2.97(0.92-9.93)$ \\
Long segment & $2.45(0.45-11.49)$ \\
\hline
\end{tabular}

HAEC Hirschsprung-associated enterocolitis, TERPT transanal endorectal pullthrough

between different pediatric surgical centres, there is a need for an objective grading system also within pediatric surgery.

In this study, we used the Clavien-Dindo grading system, as described in 2004. The system has been widely used for evaluation of post-operative complications in general surgery [5]. The grading system has been sporadically used to evaluate outcomes in pediatric surgery, for example, to identify complications related to jejunal feeding and after repair for congenital duodenal obstructions $[6,7]$.

This was a single-centre study of individuals undergoing TERPT during a 12-year period. The grading was performed retrospectively after evaluating the first 30 postoperative days according to the grading system that Clavien-Dindo described. The advantages with this system are that all possible adverse events are included. The limitations are that the Clavien-Dindo grading was done retrospectively based on data from the medical records. This causes a risk for minor events, not noted in the medical records, to be unnoticed, while more serious events would not be missed. In this study, we found that having a Clavien-Dindo complication had a significant effect on post-operative hospital stay, indicating that also minor complications have a negative impact on the post-operative care. Regarding the complications usually described in the literature as anastomotic leakage, stricture, postoperative Hirschsprung-associated enterocolities (HAEC), wound infections, bleeding, and perianal excoriations, we believe that the Clavien-Dindo grading would identify all of them except for milder perianal excoriations.

To determine whether the short-term complications differed between the genders, we performed a gender analysis to see if they were prone to different types of complications, but no significant difference was found. This result may be a type II error due to the few females included in the study.

In this study, we could not identify any risk factors for developing a Clavien-Dindo complication, although there is an obvious risk for type II errors. We know from other studies that preoperative HAEC and length of aganglionosis are factors associated with post-operative complications, although this could not be confirmed in our study [8].

We chose to exclude individuals who had other surgical procedures than TERPT to be sure to compare the post-operative courses of one procedure. Individuals with TCA were excluded, since they also undergo a more extensive surgical procedure. Individuals who did not undergo TERPT at our clinic were excluded, since we could not properly evaluate them according to Clavien-Dindo.

We could not find any patient with an anastomotic leakage. Twelve patients (17.4\%) had anastomotic strictures compared to $4-10 \%$ reported in the literature $[3,4]$. This 
is partly a definition issue, and we have defined a stricture formation as a stricture in need for dilatation under general anaesthesia. Our study also correlates well with the $1.3 \%$ infection incidence reported by Teitelbaum et al. [9]. The patients in our study had a median hospital stay of 5 days, which is similar to a multicenter study by Elhalaby et al. showing a mean hospital stay of 4.8 days [10]. Twenty-two percent of the patients had a Clavien-Dindo complication, most of them low-grade. All low-grade complications (Grade I) were related to post-operative pain, which makes it necessary to address routines for postoperative analgesia. The Grade II was connected to non-surgical event in three of the patients, while one was caused by bleeding postoperatively. The only Grade IIIb complication was directly connected to the surgical procedure. This grading system reveals more about the post-operative course compared to only reporting post-operative in hospital stay and rates of leakage, stricture, and infections. We think that the usage of an objective classification scoring system for postoperative complication would make comparisons between different centres possible and make the results transparent.

\section{Conclusion}

This is the first study to evaluate objectively post-operative complications in HSCR. Using the Clavien-Dindo grading showed that $22 \%$ of the patients had a post-operative complication, although most of them were Grade I. There is a need for further studies to evaluate objectively post-operative complications after pediatric surgical procedures.

Acknowledgements Open access funding provided by Karolinska Institute. To Eva Hagel at LIME Institution at Karolinska Institutet for all the statistical support with the study. This study was supported by the Her Royal Highness Crown Princess Lovisa's Foundation and the Sällskapet Barnavård.

\section{Compliance with ethical standards}

Conflict of interest The authors declare that they have no conflict of interest.

Ethical approval All procedures performed in studies involving human participants were in accordance with the ethical standards of the institutional and/or national research committee and with the 1964 Helsinki declaration and its later amendments or comparable ethical standards.
Informed consent Informed consent was obtained from all individual participants included in the study.

Open Access This article is distributed under the terms of the Creative Commons Attribution 4.0 International License (http://creativeco mmons.org/licenses/by/4.0/), which permits unrestricted use, distribution, and reproduction in any medium, provided you give appropriate credit to the original author(s) and the source, provide a link to the Creative Commons license, and indicate if changes were made.

\section{References}

1. Löf Granström A, Svenningsson A, Hagel E, Oddsberg J, Nordenskjöld A, Wester T (2016) Maternal risk factors and perinatal characteristics for Hirschsprung disease. Pediatrics 138(1):e20154608

2. De la Torre-Mondragón L, Ortega-Salgado JA (1998) Transanal endorectal pull-through for Hirschsprung's disease. J Pediatr Surg 33:1283-1286

3. Langer JC, Durrant AC, de la Torre L, Teitelbaum DH, Minkes RK, Caty MG et al (2003) One-stage transanal Soave pullthrough for Hirschsprung disease: a multicenter experience with 141 children. Ann Surg 238:569-583 (discussion 83-5)

4. Bjørnland K, Pakarinen MP, Stenstrøm P, Stensrud KJ, Neuvonen $M$ et al (2017) A Nordic multicenter survey of long-term bowel function after transanal endorectal pull-through in 200 patients with rectosigmoid Hirschsprung disease. Pediatr Surg 52:1458-1464

5. Dindo D, Demartines N, Clavien PA (2004) Classification of surgical complications: a new proposal with evaluation in a cohort of 6336 patients and results of a survey. Ann Surg 240(2):205-213

6. McCann C, Cullis PS, McCabe AJ, Munro FD (2019) Major complications of jejunal feeding in children. J Pediatr Surg $54: 258-262$

7. Gfroerer S, Theilen TM, Fiegel HC, Rolle U (2018) Laparoscopic versus open surgery for the repair of congenital duodenal obstructions in infants and children. Surg Endosc 32:3909-3917

8. Huang WK, Li XL, Zhang J, Zhang SC (2018) Prevalence, risk factors, and prognosis of postoperative complications after surgery for hirschsprung disease. J Gastrointest Surg 22:335-343

9. Teitelbaum DH, Cilley RE, Sherman NJ, Bliss D, Uitvlugt ND, Renaud EJ et al (2000) A decade of experience with the primary pull-through for hirschsprung disease in the newborn period: a multicenter analysis of outcomes. Ann Surg 232:372-380

10. Elhalaby EA, Hashish A, Elbarbary MM, Soliman HA, Wishahy MK, Elkholy A et al (2004) Transanal one-stage endorectal pullthrough for Hirschsprung's disease: a multicenter study. J Pediatr Surg 39:345-351 (discussion-51)

Publisher's Note Springer Nature remains neutral with regard to jurisdictional claims in published maps and institutional affiliations. 